\title{
Productivité de génotypes caprins de race pure et par croisement dans les oasis du Sud tunisien
}

\author{
A. Gaddour ${ }^{1 *}$ S. Najari ${ }^{1}$ M. Ouni ${ }^{1}$ M. Ben Hamouda ${ }^{1}$
}

Mots-clés

Caprin - Croisement - Productivité Oasis - Tunisie.

\begin{abstract}
Résumé
La comparaison des races pures caprines et des génotypes issus de croisement d'absorption de la chèvre locale par des races amélioratrices a pour but de déterminer les meilleurs génotypes caprins qui permettent la valorisation des ressources des oasis. L'étude des performances individuelles de croissance des chevreaux de la population locale, des races Alpine, Damasquine et Murciana-Granadina est insuffisante pour conclure sur l'intérêt génétique et économique lors du choix de la race amélioratrice. La présente étude a permis d'établir des indices bioéconomiques pour évaluer la productivité des groupes génétiques purs et croisés. L'indice viande a corrigé les performances de la croissance par le poids métabolique des adultes et les taux de productivité numérique. L'analyse des indices, élaborés à partir d'un fichier de données de seize campagnes de contrôles des performances, a conduit à des évaluations des génotypes assez différentes de celles établies à partir des comparaisons des performances individuelles. Il est nécessaire d'élaborer des indices plus complets, qui permettent de mieux considérer la productivité ainsi que les charges de l'élevage caprin intensif, et de les appliquer à l'ensemble des données afin de répondre aux objectifs génétiques et économiques du projet de croisement d'absorption de la population caprine locale.
\end{abstract}

\section{INTRODUCTION}

L'espèce caprine a pu, depuis sa domestication, manifester une importance économique dans les différents modes d'élevage (6), même dans les régions les plus difficiles, milieux très humides ou très arides $(10,17,18)$. Le cheptel caprin constitue une population animale rustique possédant une assez large variabilité de caractéristiques et de performances $(3,4,7,21,22)$. Après avoir joué pendant longtemps un rôle essentiel dans la vie économique et sociale de la région en l'absence d'activité concurrente, l'élevage caprin connaît actuellement un déclin important (8). Cette situation rend nécessaire et urgente l'organisation de l'élevage caprin et l'amélioration de sa rentabilité pour retrouver un essor et mieux contribuer

1. Institut des régions arides, Médenine, Tunisie.

* Auteur pour la correspondance

Tél. : +21675633005; fax: +21675633006

E-mail : gaddour.omar@yahoo.fr / amor.gaddour@ira.agrinet.tn à l'effort national de réhabilitation du secteur agricole $(14,15,19$, 20). L'amélioration de la productivité de cet élevage pourrait être envisagée à travers plusieurs voies, essentiellement par l'action sur les facteurs de production ou sur le potentiel génétique du cheptel $(2,24)$. Dans les oasis de la région, à côté de l'élevage caprin traditionnel, il existe aussi un important cheptel caprin élevé pour produire du lait dans un système d'élevage semi-intensif qui profite de l'agriculture irriguée $(9,11,16)$.

Toutefois, les productions de lait et de viande par la chèvre locale ne valorisent pas les ressources locales, tant du point de vue des facteurs de production que du mode de conduite pratiqué dans les oasis (alimentation, fourrages verts, abreuvement disponible, temps de pâturage réduit, ombrage...). Le facteur limitant l'amélioration de la productivité semblerait donc être le potentiel génétique de la chèvre locale (13). C'est la raison pour laquelle a été choisie l'option de croisements d'absorption de la chèvre locale par des races amélioratrices de potentiel génétique supérieur, pour une meilleure valorisation des ressources des oasis (23). 
Les races amélioratrices importées comprenaient l'Alpine, connue dans le monde entier pour ses hautes performances laitières, la Damasquine, spécialisée dans la production de viande, et la Murciana-Granadina qui est une race mixte (viande, lait). Ces races ont été utilisées pour la création de nouveaux génotypes plus productifs par le biais de croisement d'absorption de la chèvre locale (28). L'objectif était d'abord économique et se résumait au choix d'une race amélioratrice qui permettait la création d'un matériel génétique caprin dont la productivité dans les oasis était supérieure à celle de la population locale.

Dans la présente étude, les auteurs ont évalué la productivité des différents groupes génétiques, c'est-à-dire la population locale, les trois races importées, et les deux premières générations $\mathrm{F} 1$ et F2 des croisements d'absorption, en appliquant des corrections de performances brutes par les paramètres de reproduction et le poids métabolique adulte de chaque groupe (1). En effet, la productivité des groupes génétiques peut être estimée en tenant compte de l'efficacité de la reproduction et de la conduite, et des charges correspondant aux animaux stériles, aux avortements et au nombre des chevreaux morts avant le sevrage. Quant aux charges d'élevage, la différence principale entre les groupes génétiques peut être représentée par le poids métabolique adulte, directement proportionnel au coût de l'alimentation. Cette démarche permet d'expliciter les différences de classements établis sur les performances moyennes brutes et sur les performances corrigées (5). Le projet de croisement d'absorption de la chèvre locale, réalisé par l'Institut des régions arides de Médenine, dans le cadre d'une coopération internationale, a eu pour but de créer un nouveau matériel génétique caprin qui optimise l'utilisation des ressources disponibles dans les oasis, pour la production laitière caprine.

\section{MATERIEL ET METHODES}

\section{Collecte des données}

Les animaux ont été sujets à des contrôles périodiques et individuels de croissance pour les chevreaux. De même, les performances de reproduction (lutte, stérilité, mise bas, avortement) et les taux de mortalité ont été enregistrés régulièrement.

La base de données utilisée pour l'étude a été celle établie par l'Institut des régions arides de Médenine durant la période 1980-96. A cet égard, il faut souligner l'importance de la création d'une base pour faciliter l'accès à l'information. En effet, il a été nécessaire de regrouper plusieurs fichiers de différents formats et sources pour bénéficier au maximum des contrôles de performances réalisés. De fait, chaque chercheur disposait d'une information qui ne correspondait qu'aux thèmes qu'il traitait. La diversité des informations relatives aux animaux (alimentation, reproduction, production, hygiène...) a induit la création d'une banque de données assez complète et régulièrement mise à jour. Cette banque est par ailleurs une référence pour les organismes de recherche et de développement.

Au total, 1654 fiches de croissance des chevreaux de différents groupes génétiques ont été élaborées après vérification du ficher de base (tableau I). Les contrôles des performances ont varié avec les génotypes qui ont été différemment représentés dans le troupeau expérimental. Les données relatives aux performances de reproduction et à la mortalité des chevreaux correspondent à des informations annuelles enregistrées sur les fiches de chaque groupe génétique.

\section{Evaluation de la productivité}

L'objectif de la correction des performances est de permettre une comparaison des groupes génétiques en tenant compte de leur rentabilité économique (5). La production de viande par campagne d'élevage dépend de la reproduction, de la conduite sanitaire et de la croissance individuelle des chevreaux. L'efficacité de la reproduction peut être estimée par des paramètres comme la fertilité, qui permet de tenir compte des charges relatives aux animaux stériles, et également par les taux d'avortement et de prolificité qui sont en relation directe avec la taille de la portée (23). Enfin, le nombre des chevreaux produits et sevrés dépend également des taux de mortalité des jeunes. Le produit du nombre des chevreaux par la croissance individuelle permet d'appréhender la production de viande de chaque groupe génétique pour tous les animaux présents dans la station $(25,27)$. Dans cette étude, la correction a été faite par le poids métabolique des adultes, les paramètres de reproduction, et les taux de mortalité, sur les performances individuelles brutes des différents groupes génétiques (26). Le calcul des indices de productivité sur ces bases, détaillés précédemment, permet une comparaison des groupes génétiques plus adéquate du point de vue économique.

\section{Correction par les performances de reproduction}

\section{Indice de production de viande}

Cet indice a été estimé pour deux âges des chevreaux : à 30 et 120 jours. Il s'agissait du calcul du nombre moyen de chevreaux vifs à chaque âge par rapport à l'effectif élevé par génotype.

$\mathrm{N} 1$ = nombre de chevreaux à l'âge de 30 jours par femelle mise à la lutte $=($ taux de fécondité - taux de mortalité à 30 jours $) / 100$;

$\mathrm{N} 2$ = nombre de chevreaux à l'âge de 120 jours produits par femelle mise à la lutte $=($ taux de fécondité - taux de mortalité au sevrage) / 100 .

\section{Calcul de production de viande corrigée}

$\mathrm{V} 1$ = production de viande par femelle mise à la lutte relative aux chevreaux à l'âge de 30 jours $=\mathrm{N} 1 \mathrm{x}$ poids moyen des chevreaux à 30 jours ;

\section{Tableau I}

Récapitulatif des fiches de croissance, du nombre de campagnes de suivis des paramètres de reproduction et des taux de mortalité des chevreaux par génotype étudié

\begin{tabular}{lcc}
$\begin{array}{l}\text { Groupe } \\
\text { génétique }\end{array}$ & $\begin{array}{c}\text { Nb. chevreaux } \\
\text { soumis au contrôle } \\
\text { de croissance }\end{array}$ & $\begin{array}{c}\text { Nb. campagnes de } \\
\text { suivis des paramètres } \\
\text { zootechniques }\end{array}$ \\
\hline Locale & 148 & 12 \\
Alpine (A) & 767 & 15 \\
Damasquine (D) & 169 & 11 \\
Murciana-Granadina (M) & 148 & 13 \\
F1A & 137 & 12 \\
F1D & 49 & 8 \\
F1M & 15 & 5 \\
F2A & 176 & 8 \\
F2D & 28 & 5 \\
F2M & 17 & 2 \\
Total & 1654 & 91
\end{tabular}

F1A, F2A : croisés Alpine x locale ; F1D, F2D : croisés Damasquine x locale ; F1M, F2M : croisés Murciana-Granadina $x$ locale ; F1 : première génération de croisement ; F2 deuxième génération 
$\mathrm{V} 2$ = production de viande par femelle mise à la lutte relative aux chevreaux à l'âge de 120 jours = N2 x poids moyen des chevreaux à 120 jours.

\section{Correction par le poids métabolique}

Ces charges sont essentiellement dues à l'alimentation et sont donc directement liées au poids des brebis (5). Etant donné les différences importantes de poids adultes des différents génotypes, cette correction est apparue nécessaire pour pouvoir comparer la productivité.

P1 = quantité de la viande produite par femelle mise à la lutte relative aux chevreaux à l'âge de 30 jours = V1 / poids métabolique ;

P2 = quantité de la viande produite par femelle mise à la lutte relative aux chevreaux à l'âge de 120 jours = V2 / poids métabolique.

\section{RESULTATS ET DISCUSSION}

\section{Performances corrigées par les paramètres de reproduction}

La détermination du nombres de chevreaux par groupe génétique a été calculée par la soustraction du taux de mortalité et de fécondité (tableau II). Pour les races pures, la chèvre locale s'est distinguée par le nombre plus élevé de chevreaux produits à tous les âges étudiés. Une chèvre locale a donné, par femelle mise à la lutte, à l'âge de 30 jours en moyenne 1,41 chevreau et 1,29 chevreau atteignant l'âge de 120 jours. Pour les croisés, seuls les chevreaux F1 Damasquine $\mathrm{x}$ locale ont dépassé en nombre les chevreaux produits par la chèvre locale (tableau II).

En race pure, la chèvre Damasquine a confirmé sa réputation de race spécialisée dans la production de viande, alors que le classement de la race Alpine a changé, puisqu'elle a présenté une production de viande inférieure à celle de la population locale. Ce résultat a reflété la forte sensibilité de la race Alpine au mode de conduite du troupeau et aux aléas climatiques de la Tunisie. Seuls les croisés Damasquins (F1D et F2D) ont présenté des productions de viande supérieures à celle de la population locale, voire même à celle de la race pure (tableau III).

\section{Tableau II}

Nombre de chevreaux produits aux âges types pour chaque femelle mise à la lutte

\begin{tabular}{lcc} 
Groupe génétique & \multicolumn{2}{c}{ Nb. chevreaux } \\
\cline { 2 - 3 } & N1 & N2 \\
\hline Alpine (A) & 1,23 & 1,06 \\
Damasquine (D) & 1,28 & 1,12 \\
Murciana-Granadina (M) & 1,18 & 1,04 \\
Locale & 1,41 & 1,29 \\
F1A & 1,22 & 1,11 \\
F2A & 1,07 & 1,04 \\
F1D & 1,54 & 1,36 \\
F2D & 1,39 & 1,27 \\
F1M & 1,36 & 1,22 \\
F2M & 1,05 & 1,05
\end{tabular}

F1A, F2A : croisés Alpine x locale ; F1D, F2D : croisés Damasquine x locale ; F1M, F2M : croisés Murciana-Granadina x locale ; N1 : nombre des chevreaux à 30 jours; $\mathrm{N} 2$ : nombre des chevreaux à 120 jours; $\mathrm{F} 1:$ première génération de croisement ; F2 deuxième génération

\section{Performances corrigées par le poids métabolique}

L'objectif de cette correction a été d'inclure les charges d'alimentation relatives à chaque génotype, d'exprimer leurs différences moyennant les poids métaboliques des différents groupes génétiques (tableau IV). La chèvre locale a présenté l'indice P1 le plus élevé par rapport aux races pures importées. La productivité du génotype F1 Damasquine a été la plus élevée de tous les groupes

\section{Tableau III}

Quantité de viande produite par femelle mise à la lutte

\begin{tabular}{lcccc}
$\begin{array}{l}\text { Groupe } \\
\text { génétique }\end{array}$ & $\begin{array}{c}\text { Poids moyen } \\
\text { des chevreaux } \\
\text { (kg) }\end{array}$ & \multicolumn{2}{c}{$\begin{array}{c}\text { Quantité de } \\
\text { viande produite } \\
\text { (kg) }\end{array}$} \\
\cline { 2 - 3 } & P30 & P120 & V1 & V2 \\
\hline Alpine (A) & 8,02 & 14,81 & 9,99 & 15,7 \\
Damasquine (D) & 8,1 & 16,48 & 10,49 & 18,46 \\
Murciana- & 6,36 & 11,99 & 7,49 & 12,47 \\
Granadina (M) & & & & \\
Locale & 6,69 & 12,85 & 9,44 & 16,06 \\
F1A & 7,98 & 14,98 & 9,95 & 15,85 \\
F2A & 7,93 & 15,78 & 9,15 & 16,41 \\
F1D & 7,57 & 14,39 & 11,99 & 19,57 \\
F2D & 7,89 & 15,78 & 11,48 & 20,05 \\
F1M & 6,47 & 14,42 & 8,80 & 17,59 \\
F2M & 7,21 & 13,92 & 7,57 & 14,62 \\
& & & &
\end{tabular}

F1A, F2A : croisés Alpine x locale ; F1D, F2D : croisés Damasquine x locale ; F1M, F2M : croisés Murciana-Granadina x locale ; P30 : poids à 30 jours ; P120 poids à 120 jours ; V1 : quantité de la viande produite à 30 jours ; V2 : quantité de la viande produite à 120 jours ; F1 : première génération de croisement ; F2 deuxième génération

\section{Tableau IV}

Quantité de viande produite pour un kilogramme de poids métabolique de femelle mise à la lutte

\begin{tabular}{lcc}
$\begin{array}{l}\text { Groupe } \\
\text { génétique }\end{array}$ & $\begin{array}{l}\text { Quantité de viande produite (kg) } \\
\text { pour 1 kg du poids métabolique }\end{array}$ \\
& P1 & P2 \\
\hline Alpine (A) & 0,52 & 0,82 \\
Damasquine (D) & 0,58 & 1,03 \\
Murciana-Granadina (M) & 0,54 & 0,9 \\
Locale & 0,71 & 1,21 \\
F1A & 0,67 & 1,07 \\
F2A & 0,54 & 0,96 \\
F1D & 0,76 & 1,25 \\
F2D & 0,73 & 1,28 \\
F1M & 0,65 & 1,27 \\
F2M & 0,55 & 1,06
\end{tabular}

F1A, F2A : croisés Alpine x locale ; F1D, F2D : croisés Damasquine x locale ; F1M, F2M : croisés Murciana-Granadina x locale ; P1 : quantité de la viande produite pour $1 \mathrm{~kg}$ de poids métabolique à 30 jours ; P2 : quantité de la viande produite pour $1 \mathrm{~kg}$ de poids métabolique à 120 jours ; F1 : première génération de croisement ; F2 deuxième génération. 
génétiques présents, et la lignée F1 Murciana-Granadina a présenté un rendement supérieur à celui de l'Alpine et de ses croisements. Toutefois, la différence entre le rendement du groupe génétique F1 Damasquine et la population locale a été assez faible (environ $0,05 \mathrm{~kg}$ pour $1 \mathrm{~kg}$ du poids métabolique), ce qui n'a pas permis de préconiser son élevage.

\section{- CONCLUSION}

L'évaluation de la productivité des groupes génétiques purs et croisés a conduit à des résultats différents selon l'approche de la comparaison. En effet, l'usage des indices bioéconomiques a donné lieu à des classifications des groupes génétiques différentes de celles issues des études de performances individuelles. Par conséquent, l'intégration des composantes de la productivité, telles que la fertilité, la mortalité et le poids métabolique, est indispensable pour un choix raisonné d'une race amélioratrice. Pour la production de viande, la race Damasquine et la population locale ont permis les meilleurs nivaux de productivité. Toutefois, l'élaboration des indices bioéconomiques doit tenir compte de toutes les composantes et des facteurs de production pour aboutir à des évaluations conformes aux objectifs économiques et zootechniques de croisement d'absorption de la population locale.

\section{BIBLIOGRAPHIE}

1. AKINSOYINU A.O., MBA A.U., OLUBAJO F.O., 1997. Studies on milk yield and composition of the West African Dwarf goat in Nigeria. J. Dairy Res., 44: 57-62.

2. ALEXANDRE G., AUMONT G., DESPOIS P., .MAINAUD J.C., COPPRY O., XANDE A., 1997. Productive performances of Guadeloupean Creole goats during the suckling period. Small Rumin. Res., 34: 157-162.

3. BAGHEL M.S., GUPTA M.P., 1979. Nitrogen distribution in goat milk, J. Dairy Sci., 32: 340-342.

4. BOYAZOGLU J., MORAND-FEHR P., 2001. Mediterranean dairy sheep and goat products and their quality. A critical review. Small Rumin. Res. 40: $1-11$.

5. CAJA G., 1990. Evolution des systèmes de production ovin-lait dans le Bassin méditerranéen. Options Méditerr., 12 : 31-38.

6. CHEMINEAU P., MAHIEU M., VARO H., SHITALOU E., JEGO Y., GRUDE A., THIMONIER J., 1991. Reproduction des caprins et des ovins Créoles de Guadeloupe et de Martinique. Revue Elev. Méd. vét. Pays trop., $44:$ 45-50.

7. DE CREMOUX R., 1995. Relations entre les numérations cellulaires du lait et les infections mammaires chez la chèvre. Thèse Doct. vét., Ecole nationale vétérinaire, Toulouse, France, $71 \mathrm{p}$.

8. DEVENDRA C., MC LEROY G.B., 1982. Goat and sheep production in the tropics. In: Proc. 7th International Tropical Agricultural Series. London, UK, Longman, $271 \mathrm{p}$.

9. GADDOUR A., 2005. Performances de croissance et de production laitière des groupes génétiques caprins issus d'un croisement d'absorption de la chèvre locale dans les oasis du sud tunisien. Mastère Génét. Bio Ressources, faculté des Sciences, Tunis, Tunisie, $73 \mathrm{p}$.

10. GADDOUR A., NAJARI S., 2008. Adjustment of the kid's growth curve in pure goat breeds and crosses under southern Tunisian conditions. J. Appl. Anim. Res., 2: 117-120.

11. GADDOUR A., NAJARI S., ABDENNEBI M., OUNI M., 2007. Reproductive performances and kid's mortality of pure breeds and crossed caprine genotypes in the coastal oases of southern Tunisia. Pak. J. biol. Sci., 10: 2314-2319.

12. GADDOUR A., NAJARI S., FERCHICHI A., 2009. Milk production of caprine genotypes in arid land of southern Tunisia. Res. J. Dairy Sci., 3: 1-2.

13. GADDOUR A., NAJARI S., FERCHICHI A., ABDENNEBI M., 2009. Lactation curve of local goat, pure breds and crosses genotypes in Southern Tunisia. J. Appl. Anim. Res., 36.

14. GADDOUR A., NAJARI S., OUNI M., 2007. Dairy performances of the goat genetic groups in the southern Tunisian. Agric. J., 2: 248-253.
15. GADDOUR A., NAJARI S., OUNI M., 2007. Kid's growth of pure breeds and crossed caprine genotypes in the coastal oases of southern Tunisia. Res. J. Agron., 2: 51-58.

16. GADDOUR A., NAJARI S., OUNI M., 2007. Kid's growth and dairy performances of pure breds and crossed caprine genotypes in the coastal oases of southern Tunisia. Pak. J. biol. Sci., 10: 2874-2879.

17. GADDOUR A., NAJARI S., OUNI M., 2008. Amélioration de la production laitière caprine par le croisement d'absorption dans une oasis du Sud tunisien. Revue Elev. Méd. vét. Pays trop., 61 : 57-62.

18. GADDOUR A., NAJAR S., OUNI M., 2008. Productive performances of pure breds and crossed caprine genotypes in the southern Tunisia. Options Méditerr., Ser. A (79): 234-238.

19. GADDOUR A., NAJARI S., OUNI M., 2008. The genotypeenvironment interaction effects on dairy performances of goat genetic groups in the Tunisian oases. Res. J. Dairy Sci., 1: 22-26.

20. GADDOUR A., OUNI M., ABDENNABI M., NAJARI S., 2008. Valorisation des ressources oasiennes par l'intensification de la production caprine (Capra hircus). Revue Régions arides, 21 ( ${ }^{\circ}$ spécial) : 1331-1339.

21. HAENLEIN G.F.W., 1992. Role of goat meat and milk in human nutrition. In: Proc. 5th Int. Conf. Goats, New Delhi, India, 1-8 March 1991, Vol. II, part II. New Delhi, India, ICAR, p. 575-580.

22. JASH S., SINGH C., GUPTA A.K., 2001. Effect of enhanced prepartum concentrate feeding on kidding and lactation performance of stallfed crossbred goats. Indian J. Small Ruminants, 7: 19-24.

23. LE GAL O., PLANCHENAULT D., 1993. Utilisation des races caprines dans les zones chaudes. Contraintes et intérêts. Maisons-Alfort, France, Cirad-emvt, $261 \mathrm{p}$.

24. MORAND-FEHR P., BOUTONNET DEVENDRA C., DUBEUF HAENLEIN G.F.W., HOLST P., CAPOTE J., 2004. Strategy for goat farming in the 21st century. Small Ruminant Res., 51: 175-183.

25. NAJARI S., 2005. Caractérisation zootechnique et génétique d'une population caprine. Cas de la population caprine locale des régions arides tunisiennes. Thèse Doct. Etat, Institut national agronomique, Tunis, Tunisie, $214 \mathrm{p}$.

26. NAJARI S., GADDOUR A., ABDENNEBI M., BEN HAMOUDA M., KHALDI G., 2006. Caractérisation morphologique de la population caprine locale des régions arides tunisiennes, Revue Régions arides, 17 : 23-41.

27. NAJARI S., GADDOUR A., BEN HAMOUDA M., DJEMALI M., KHALDI G., 2007. Growth model adjustment of local goat population under pastoral conditions in a Tunisian arid zone. J. Agron., 6: 61-67.

Accepté le 05.02.2010 


\section{Summary}

Gaddour A., Najari S., Ouni M., Ben Hamouda M. Productivity of Pure Breeds and Crossed Caprine Genotypes in the Coastal Oases of Southern Tunisia

The comparison of pure breeds of goats and genotypes resulting from crossing of absorption of the local goat by ameliorative breeds aims at determining the best goat genotypes which allow enhancing oasis resources. The study of individual growth performances in kids of the local population, in Alpine, Damascus and Murciana-Granadina breeds is insufficient to conclude on genetic and economic advantages allowing to select the ameliorative breed. This study helped to establish bioeconomic indices to evaluate the productivity of pure and crossed genetic groups. The meat index corrected the growth performances by the metabolic weight of the adults and productivity rates. The index analysis, elaborated from a data bank based on sixteen performance control campaigns, led to somewhat different results from those established on the comparison of individual performances. It is necessary to develop more complete indices, which will help to consider productivity better as well as costs of intensive farming, and to apply them to the whole of the data in order to answer the genetic and economic objectives of the project on crossing of absorption of the local goat population.

Keywords: Goat - Crossbreeding - Productivity - Oasis Tunisia.

\section{Resumen}

Gaddour A., Najari S., Ouni M., Ben Hamouda M. Productividad de los genotipos caprinos de raza pura y mediante cruces en los oasis del Sur tunecino

La comparación de las razas caprinas puras y de los genotipos originados de cruces cuyo fin es la absorción de la cabra local por razas mejoradoras tiene como objetivo el de determinar los mejores genotipos caprinos que permitan la valorización de los recursos del oasis. El estudio de los rendimientos individuales de crecimiento de los cabritos de la población local, de razas Alpina, Damasquina y de Murciana-Granadina es insuficiente para concluir sobre el interés genético y económico en el momento de escoger la raza mejoradora. El presente estudio permitió establecer los índices bio económicos para evaluar la productividad de los grupos genéticos puros y cruzados. El índice de carne corrigió los rendimientos de crecimiento por peso metabólico de los adultos y las tasas de productividad numérica. El análisis de los índices, elaborados a partir de una base de datos de dieciséis campañas de control de los rendimientos, condujo a evaluaciones de los genotipos bastante diferentes de aquellas establecidas a partir de comparaciones individuales. Es necesario elaborar índices más completos, que permitan una mejor consideración de la productividad, así como de las cargas de cría caprina intensiva y aplicarlas al conjunto de los datos con el fin de responder a los objetivos genéticos y económicos del proyecto de cruces de absorción de la población caprina local.

Palabras clave: Caprino - Cruzamiento - Productividad Oasis - Túnez. 